(2) Open Access Full Text Article

\title{
Association Between the Cardiometabolic Index and Hyperuricemia in an Asymptomatic Population with Normal Body Mass Index
}

\author{
Yu-Qiang Zuo (D)' \\ Zhi-Hong Gao' \\ Yu-Ling Yin' \\ Xu Yang' \\ Ping-Yong Feng ${ }^{2}$ \\ 'Department of Health Physical \\ Examination Center, The 2nd Hospital of \\ Hebei Medical University, Shijiazhuang, \\ People's Republic of China; ${ }^{2}$ Department \\ of Imaging Center, The 2nd Hospital of \\ Hebei Medical University, Shijiazhuang, \\ People's Republic of China
}

Background and Purpose: The association between the cardiometabolic index (CMI) and hyperuricemia was investigated to provide theoretical support for the management of hyperuricemia in an asymptomatic population with normal body mass index (BMI).

Methods: A cross-sectional study was carried out among 374 asymptomatic adults with normal BMI. Traditional anthropometric indices and CMI were calculated. Anthropometric indices were divided into four quartiles and multivariate logistic analysis was used to analyze the association between these indices and hyperuricemia. Receiver operating characteristic (ROC) curves and area under the curve (AUC) were used to evaluate the power of the indices to predict hyperuricemia values. The DeLong test was used to compare the AUC of different anthropometric indices.

Results: After adjusting for confounding variables, the CMI exhibited a stronger association with hyperuricemia than other anthropometric indices. The odds ratio (OR) for hyperuricemia in the highest quartile of the CMI was 16.674 (confidence interval $[\mathrm{CI}]=4.424-62.846$ ). The AUC of the CMI was 0.777 (95\% CI $=0.719-0.835, p<0.001)$, which was higher than the values for other anthropometric indices. The differences in AUC between the CMI and other indices were statistically significant; the optimal cutoff value of the CMI was 0.655 , with sensitivity of $57.1 \%$ and specificity of $84.2 \%$.

Conclusion: The CMI, which combines waist circumference, height and blood lipid parameters, was more strongly associated with hyperuricemia than other anthropometric indices in asymptomatic population with normal BMI. The CMI may serve as a potential monitoring indicator for hyperuricemia management in asymptomatic populations with normal BMI.

Keywords: cardiometabolic index, hyperuricemia, visceral adiposity index, lipid accumulation product

\section{Introduction}

Hyperuricemia is highly prevalent worldwide, as previously reported for random general populations, ranging from $13.2 \%$ in China to $21.2 \%$ in the US. ${ }^{1,2}$ Hyperuricemia not only contributes to gout and nephrolithiasis but is also related to metabolic syndrome (MS), cardiovascular disease, and non-alcoholic fatty liver disease. There are many factors related to the pathogenesis of hyperuricemia, with obesity considered to be a particularly important factor, so recommendations for the management of hyperuricemia have typically emphasized the beneficial effects of weight loss. ${ }^{3}$ However, there are still many asymptomatic adults with normal body mass index (BMI) who have hyperuricemia. Therefore, the development of accurate
Correspondence: Ping-Yong Feng Email xjfpy@I63.com 
and cost-efficient screening tools for hyperuricemia in the population with normal BMI is urgently needed.

There are many anthropometric indices which have been developed to evaluate adiposity of the body, such as BMI, which is adjusted by height and body weight and can evaluate general adiposity but cannot differentiate between muscle and adipose tissue mass. In recent years, researchers have found that central obesity is more strongly associated with health status, so other measures have been proposed to evaluate abdomen adipose tissue including hip circumference (HC), waist circumference (WC), waist to hip ratio (WHR), waist height ratio (WHtR), a body shape index (ABSI), ${ }^{4}$ body adiposity index (BAI) ${ }^{5}$ and body roundness index (BRI). ${ }^{6}$ Some studies found that these indices were more strongly associated with metabolic abnormality than BMI., However, these indices can still not exactly differentiate visceral adipose tissue from subcutaneous adipose tissue, and for this reason, the visceral adiposity index (VAI) ${ }^{9}$ was introduced to evaluate the distribution of visceral adiposity. Some studies ${ }^{10,11}$ have reported that these adiposity indices can help to evaluate MS more accurately, including diabetes mellitus, hyperlipidemia, and hyperuricemias, especially for obese adults. In 2006, the lipid accumulation product (LAP) ${ }^{12}$ was introduced, which incorporated triglycerides (TG) and $\mathrm{WC}$ and was adjusted by gender, which was thought to offer a better evaluation of visceral adipose tissue and had a stronger association with metabolic status. Some studies ${ }^{13,14}$ reported that LAP was an efficient marker of MS in general adult populations. In 2015, the cardiometabolic index $(\mathrm{CMI})^{15}$ was proposed as a new index to evaluate visceral obesity by using blood lipid parameters and WHtR. The CMI has been verified to be associated with a variety of metabolic diseases, and it also provides a higher predictive value for hyperuricemia in random populations compared to other traditional anthropometric indices. ${ }^{3}$ However, whether the CMI is associated with hyperuricemia in asymptomatic adults with normal BMI has not been reported to the best of our knowledge, and it is also unknown if the CMI provides higher predictive value for hyperuricemia compared to other anthropometric indices in asymptomatic adults with normal BMI. Therefore, we designed this cross-sectional study to compare the association between these anthropometric indices and hyperuricemia, which may provide a theoretical basis for management of asymptomatic hyperuricemia adults with normal BMI.

\section{Materials and Methods}

\section{Participants}

This study was a cross-sectional analysis. Participant inclusion criteria were (1)asymptomatic adults over 18 years old; (2) complete data available for anthropometric indices; (3)complete laboratory data available for blood lipid and related biomarkers; (4) according to the Chinese obesity BMI standard, the normal BMI range is: $18.5 \leq \mathrm{BMI}<24 \mathrm{Kg} / \mathrm{m}^{2}$. Exclusion criteria were (1)current use of medicine for lowering serum uric acid (SUA) or regulating lipids; (2)presence of clinical symptoms related to hyperuricemia; (3) chronic kidney disease or impaired renal function; (4)participants with malignancies; (5)participants with cardiovascular events, autoimmune diseases which may affect the SUA level. The cross-sectional study was conducted during routine health check-up examinations on 374 consecutively enrolled participants from January 2021 to July 2021, and the clinical data were collected. The study protocol complies with the Declaration of Helsinki, and ethical approval was obtained from the second hospital of Hebei Medical University hospital ethics committee (approval No.2021-P019).

\section{Anthropometric and Biochemical Measurements}

The height and weight of participants were measured with electronic scales when barefoot wearing light clothes. WC and $\mathrm{HC}$ were measured by trained nurses with inelastic tape; the WC was measured at the middle level of the lower costal rib and top of the iliac crest, and $\mathrm{HC}$ was obtained at the point of maximum circumference of the buttocks. Blood pressure (systolic blood pressure and diastolic blood pressure) were measured via automatic blood pressure monitor (HEM-7132, Omron), every participant took twice with an interval of 5 minutes, and the mean value of the twice as the final value. The blood samples of participants were collected after more than $8 \mathrm{~h}$ of overnight fasting. Fasting plasma glucose (FPG), serum urea, serum creatinine (SCr), SUA, total cholesterol (TC), triglycerides (TG), high-density lipoprotein cholesterol (HDL-C) and low-density lipoprotein cholesterol (LDL-C) were measured by biochemical autoanalyzer (Beckman Coulter AU5800, Tokyo, Japan).

\section{Definitions and Anthropometric Indices}

\section{Calculations}

Hyperuricemia diagnosis was $\mathrm{SUA}>420 \mu \mathrm{mol} / \mathrm{L} .{ }^{16}$ Anthropometric indices were calculated by the following 
formulas: $\quad \mathrm{WHR}=\mathrm{WC}(\mathrm{cm}) / \mathrm{HC}(\mathrm{cm}) ; \quad \mathrm{WHtR}=\mathrm{WC}(\mathrm{cm}) /$ Height $(\mathrm{cm}) ; \quad$ ABSI $=\mathrm{WC}(\mathrm{cm}) /[\text { height }(\mathrm{cm})]^{1 / 2} *\left(\mathrm{BMI}^{2}\right)^{1 / 3}$; $\mathrm{BAI}=\mathrm{HC}(\mathrm{cm}) / \mathrm{H}(\mathrm{m})^{2 / 3}-18 ; \mathrm{BRI}=364.2-365.5 *[1-[(\mathrm{WC} / 2 \pi) /$ $\left(0.5^{*}\right.$ height $\left.\left.)\right]^{2}\right]^{1 / 2} ; \quad \mathrm{VAI}($ males $)=\left[\mathrm{WC}(\mathrm{cm}) / 39.68+\left(1.88^{*}\right.\right.$ $\mathrm{BMI})]^{*}[\mathrm{TG}(\mathrm{mmol} / \mathrm{L}) / 1.03] *[1.31 / \mathrm{HDL}-\mathrm{C}(\mathrm{mmol} / \mathrm{L})] ; \quad \mathrm{VAI}($ females $)=[\mathrm{WC}(\mathrm{cm}) / 36.58+(1.89 * \mathrm{BMI})]^{*}[\mathrm{TG}(\mathrm{mmol} / \mathrm{L}) / 0.81$ ]*[1.52/HDL-C(mmol/L)]; CMI=TG/HDL-C*WHtR; LAP $($ females $)=\mathrm{TG}(\mathrm{mmol} / \mathrm{L}) *[\mathrm{~W}(\mathrm{~cm})-58] ; \quad \mathrm{LAP}($ males $)=\mathrm{TG}$ $(\mathrm{mmol} / \mathrm{L})^{*}[\mathrm{WC}(\mathrm{cm})-65]$. Sample size calculation in this test was based on the ability to detect the hyperuricemia at least of 0.75 (probability of type I error $=0.01$, power $=0.99$ ).

\section{Statistical Analysis}

Categorical variables are presented as numbers (percentages; $n, \%)$ and were compared with the Chi-square $\left(\chi^{2}\right)$ test. The normality of continuous variables was analyzed by the Kolmogorov-Smirnov test. Normally distributed continuous data are presented as means \pm standard deviation $(\bar{\chi} \pm s)$ or as medians with interquartile range $(\mathrm{M}$, IQR) according to whether they conformed to a normal distribution. Parametric variables were analyzed using independent $t$ tests, while nonparametric variables were analyzed using the Mann-Whitney $U$-test. The correlations between different anthropometric indices and SUA level were assessed using partial correlation analysis. The $\chi^{2}$ tests were used to compare the incidence among different quartiles of anthropometric indices. Logistic analysis was used to analyze the association between different anthropometric indices and hyperuricemia. A receiver operating characteristic (ROC) curve was built for each variable in a univariate manner, and optimal thresholds were determined based on the sensitivity and specificity. Statistical analyses were performed using SPSS software (version 25.0, IBM Corp., Armonk, NY, USA) and SAS software (Version 9.4, SAS Institute Inc., Cary, NC, USA). Statistical charts were drawn using GraphPad Prism software (version 8.0, GraphPad, Inc., La Jolla, CA, USA). Comparison of ROC curves were performed using MedCalc software (MedCalc Software Ltd., Ostend, Belgium). Statistical significance was set at $p<0.05$.

\section{Results}

\section{Basic Characteristics of Participants}

There were 374 participants enrolled in this study, including 144 men and 230 women, age range 21-80 years (41.55 \pm 12.70 years), BMI range $18.6-23.9 \mathrm{Kg} / \mathrm{m}^{2}(21.55$ $\pm 1.49 \mathrm{Kg} / \mathrm{m}^{2}$ ). The basic characteristics for all participants are summarized in Table 1. The prevalence of hyperuricemia in asymptomatic adults was $18.72 \%$ (70/374). Compared with participants with normal SUA levels, the participants with hyperuricemia had significantly higher levels of VAI, CMI, FPG, serum urea, SCr and TG, and significantly lower levels of HDL-C than normal SUA participants (all $p<0.05$ ). Differences in other anthropometric indices and biomarkers between the normal SUA and hyperuricemia group were not significant (all $p>0.05$ ).

After controlling for age, partial correlation analysis found that LAP, VAI and CMI were positively correlated with SUA level $(\mathrm{r}=0.129,0.165$ and 0.425 , respectively; all $p<0.05)$. Other anthropometric indices were not significantly correlated with SUA level $(p>0.05)$. Results of the partial correlation analysis between different anthropometric indices and SUA level are shown in Table 2.

Table 3 show the incidence of hyperuricemia in quartiles of VAI, LAP and CMI scores. The incidence of hyperuricemia increased per quartile for the CMI, and the Chi-square test was significant $\left(\chi^{2}=56.313, p<0.001\right)$ Figure 1.

After fully adjusting for age, gender, BMI and select biochemical indicators in model 3, only CMI was significantly associated with hyperuricemia $(p<0.05)$, while other anthropometric indices were without significant association with hyperuricemia (all $p>0.05$ ). Results are shown in Table 4.

The ROC curves of different anthropometric indices for hyperuricemia are shown in Table 5 and Figure 2. Among the anthropometric indices, the CMI had the best predictive value for hyperuricemia, with AUC of 0.777 (95\% CI $=0.719-0.835 ; \quad p<0.001)$, with sensitivity of $57.1 \%$ and specificity of $84.2 \%$; meanwhile, VAI had a relatively high AUC of 0.592 (95\% CI=0.525-0.658; $p<0.05$ ), with sensitivity of $85.7 \%$ and specificity of $35.4 \%$. The predictive values of ABSI, BAI, BRI and LAP were not statistically significant (all $p>0.05$ ). DeLong tests showed that the differences in AUC comparing CMI with LAP, VAI, ABSI, BAI and BRI were all significantly different $(Z=4.361,5.699,5.696,5.357$, and 5.254, respectively; all $p<0.0001)$.

\section{Discussion}

To the best of our knowledge, this is the first cross-sectional study comparing the association between different anthropometric indices and hyperuricemia in asymptomatic adults with normal BMI. The results showed that the CMI had a stronger positive relationship with hyperuricemia than did 
Table I Basic Characteristics of the Participants

\begin{tabular}{|c|c|c|c|c|}
\hline Variable & Normal SUA & Hyperuricemia & $\chi^{2} / t / z$ & $p$ value \\
\hline No. & 304 & 70 & & \\
\hline Males, n (\%) & $116(38.16)$ & $28(40.00)$ & 0.082 & $0.775^{\mathrm{a}}$ \\
\hline Age, year & $44.00,22.25$ & $37.00,19.50$ & -0.112 & $0.911^{\mathrm{b}}$ \\
\hline $\mathrm{HC}(\mathrm{cm})$ & $90.11 \pm 3.30$ & $89.68 \pm 3.19$ & 0.998 & $0.319^{c}$ \\
\hline$W C(\mathrm{~cm})$ & $79.40,6.55$ & $78.30,8.08$ & -1.001 & $0.317^{b}$ \\
\hline $\operatorname{BMI}\left(\mathrm{Kg} / \mathrm{m}^{2}\right)$ & $22.20,2.25$ & $21.15,2.75$ & -1.662 & $0.097^{\mathrm{b}}$ \\
\hline WHR & $0.88,0.05$ & $0.86,0.06$ & -0.376 & $0.707^{\mathrm{b}}$ \\
\hline $\mathrm{WHtR}$ & $0.48,0.05$ & $0.47,0.05$ & -0.552 & $0.58 \mathrm{I}^{\mathrm{b}}$ \\
\hline ABSI & $0.79,0.03$ & $0.78,0.03$ & -0.234 & $0.815^{b}$ \\
\hline $\mathrm{BAI}$ & $24.62 \pm 2.15$ & $24.50 \pm 1.98$ & 0.412 & $0.68 I^{c}$ \\
\hline BRI & $3.00,0.85$ & $2.90,0.90$ & -0.426 & $0.670^{\mathrm{b}}$ \\
\hline VAI & $1.00,1.00$ & $1.50,1.13$ & -2.312 & $0.021^{b}$ \\
\hline LAP & $21.25,17.98$ & $19.47,17.38$ & -0.019 & $0.985^{b}$ \\
\hline CMI & $0.34,0.30$ & $0.7 \mathrm{I}, 0.66$ & -7.234 & $<0.001^{b}$ \\
\hline $\mathrm{FPG}(\mathrm{mmol} / \mathrm{L})$ & $4.90,0.70$ & $5.15,1.22$ & -3.140 & $0.002^{b}$ \\
\hline Urea $(\mathrm{mmol} / \mathrm{L})$ & $4.70,1.80$ & $5.10,1.65$ & -3.429 & $0.00 \mathrm{I}^{\mathrm{b}}$ \\
\hline $\mathrm{SCr}(\mu \mathrm{mol} / \mathrm{L})$ & $63.00,16.50$ & $74.50,19.50$ & -6.876 & $<0.001^{\mathrm{b}}$ \\
\hline TC $(\mathrm{mmol} / \mathrm{L})$ & $4.53,1.22$ & $4.70,1.67$ & -1.510 & $0.131^{b}$ \\
\hline $\mathrm{TG}(\mathrm{mmol} / \mathrm{L})$ & $0.97,0.70$ & $1.55,1.38$ & -6.634 & $<0.001^{b}$ \\
\hline HDL-C (mmol/L) & I.36, 0.34 & $1.16,0.31$ & $-5.67 \mid$ & $<0.001^{\mathrm{b}}$ \\
\hline LDL-C (mmol/L) & $2.96,0.97$ & $3.27,1.26$ & -1.804 & $0.074^{b}$ \\
\hline Diabetes, n (\%) & $14(4.61)$ & $7(10.0)$ & 3.125 & $0.077^{\mathrm{a}}$ \\
\hline Current Smoking n (\%) & 48 (I5.79) & $15(21.43)$ & 1.292 & $0.256^{\mathrm{a}}$ \\
\hline Drink, n (\%) & $104(46.38)$ & $40(57.14)$ & 2.638 & $0.104^{\mathrm{a}}$ \\
\hline $\mathrm{DBP}(\mathrm{mmHg})$ & $83.00,8.25$ & $83.00,11.25$ & -1.948 & $0.05 \mathrm{I}^{\mathrm{b}}$ \\
\hline $\mathrm{SBP}(\mathrm{mmHg})$ & $127.00,13.25$ & $123.50,17.00$ & -1.600 & $0.110^{\mathrm{b}}$ \\
\hline Hypertension, n (\%) & $57(18.75)$ & $19(27.14)$ & 2.475 & $0.116^{\mathrm{a}}$ \\
\hline
\end{tabular}

Notes: ${ }^{\mathrm{a} C h i}$-square test; ${ }^{\mathrm{b}}$ Mann-Whitney U-test; ${ }^{\mathrm{C}}$ Independent $t$ test.

Abbreviations: HC, hip circumference; WC, waist circumference; WHR, waist to hip ratio; WHtR, waist height ratio; $A B S I$, a body shape index; BAI, body adiposity index; Bri, Body roundness index; VAI, visceral adiposity index; FPG, fasting plasma glucose; urea, serum urea; SCr, serum creatinine; SUA, serum uric acid; TC, total cholesterol; TG, Triglyceride; HDL-C, high-density lipoprotein cholesterol; LDL-C, low-density lipoprotein cholesterol. SBP, systolic blood pressure; DBP, diastolic blood pressure.

Table 2 Partial Correlation Analysis of Different Anthropometric Indices and SUA Level

\begin{tabular}{|l|c|c|}
\hline Anthropometric Indices & $\mathbf{r}$ & $\boldsymbol{p}$ value \\
\hline ABSI & 0.027 & 0.598 \\
BAI & -0.047 & 0.369 \\
BRI & -0.067 & 0.200 \\
VAI & 0.165 & 0.001 \\
LAP & 0.129 & 0.013 \\
CMI & 0.425 & $<0.001$ \\
\hline
\end{tabular}

other anthropometric indices even after they were fully adjusted. This result should provide new ideas for the management of SUA levels in asymptomatic adults with normal BMI.

Some studies have confirmed that the increase in adiposity, especially visceral adiposity, has a positive association with hyperuricemia. ${ }^{3}$ So, such as ABSI, BAI and BRI
Table 3 Incidence of Hyperuricemia in Quartiles of VAI, LAP and CMI

\begin{tabular}{|l|c|c|c|c|}
\hline Quartile & QI & Q2 & Q3 & Q4 \\
\hline VAI: n (\%) & $9(9.38)$ & $21(21.21)$ & $21(24.42)$ & $19(20.43)$ \\
LAP: n (\%) & $17(18.09)$ & $19(20.21)$ & $15(16.13)$ & $19(20.43)$ \\
CMI: n (\%) & $3(3.06)$ & $10(11.11)$ & $17(18.09)$ & $40(43.48)$ \\
\hline
\end{tabular}

Notes: Q1, the lowest quartile, Q2, the lower quartile, Q3, the upper quartile, Q4, the highest quartile.

new anthropometric had been proposed to distribute adipose tissue more precisely, and some studies reported that these indices have close relationship with hyperuricemia in the general population. ${ }^{17,18}$ Contrary to previous studies, we did not find any relationship between these indices and hyperuricemia in our study. The reason for these inconsistent results may be due to: (1) participants from different region with different lifestyle and BMI. (2) The BAI, ABSI 


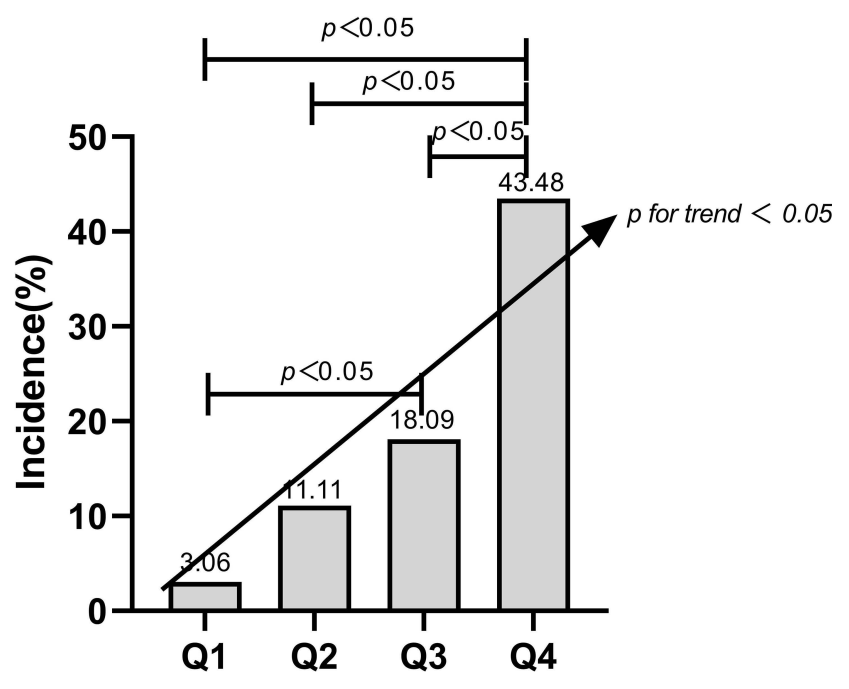

Figure I The incidence of hyperuricemia in per quartile for the CMI, and the Chisquare test results.

and BRI cannot exactly distinguish subcutaneous adipose tissue from visceral adipose tissue, the effects of them on health-related risk factors are different. ${ }^{19,20}$

The new indicators of abdominal obesity (VAI, LAP and $\mathrm{CMI}$ ), besides $\mathrm{WC}$ include lipid parameters (TG or TG/HDL-C) to better discriminate cardiometabolic risk and MS than traditional indices. ${ }^{21,22}$ Previous studies had indicated that TG/HDL-C was superior in identify MS than nonHDL-C based on multiethnic sample, ${ }^{23}$ and TG/ HDL-C considered as a marker of insulin resistance (IR) even in normal-weight adults. ${ }^{24}$ So, both the CMI and VAI have close relationship with the IR - central component of MS, and oxidative stress/inflammation-the key features of MS. The oxidative stress can induce IR by impairing insulin signal transduction and causing adipokine dysregulation, in addition, it also can activates several serinethreonine kinase pathway, such as IKK $\beta / \mathrm{NF}-\kappa \mathrm{B}$ and JNK, which leading to IR sensitive degradation and resulted to the MS finally. ${ }^{25}$ Many inflammatory mediators, particularly tumor necrosis factor- $\alpha$ (TNF- $\alpha)$, monocyte chemotactic protein-1 (MCP-1), C-reactive protein (CRP) and interleukins are upregulated in IR via serine phosphorylation of IRS-1 and it reduces GLUT-4 expression thus decreasing glucose entry into cells or inhibition of the IKK $\beta$ signaling pathway, which can improve insulin sensitivity. $^{26}$

Some scholars have reported a positive correlation between VAI and hyperuricemia, ${ }^{27,28}$ and they argue that visceral adipose tissue increase not only produce large numbers of free fatty acids which accelerate purine and uric acid synthesis, but also can cause abnormalities in peroxisomal proliferator-activated receptor- $\gamma$, resistin, cytokines and inflammatory factors, which contribute to endocrine dysfunction of adipose tissue and finally result in IR cause hyperuricemia. $^{29,30}$ But in recent years, many scholars found that the IR and hyperuricemia were mutual causal relationship, even sometimes the hyperuricemia prior the IR occurs, IR enhances renal urate reabsorption via stimulation of uric acid transporter 1 and/or the $\mathrm{Na}$-dependent anion co-transporter in brush border membranes or the renal proximal tubule; ${ }^{31,32}$ and IR accelerates the rate of lipolysis and decreases adipose tissue lipoprotein lipase activity, ${ }^{33}$ which in turn causes hyperlipemia and the subsequent overproduction of uric acid; ${ }^{34}$ on the contrary, the hyperuricemia can induce endothelial dysfunction and/or oxidative changes in adipocytes, which are typical stigmata of IR; ${ }^{35,36}$ In this study, VAI was associated with SUA level, but when fully adjusted for age, gender, BMI and biomarkers, the association with hyperuricemia disappeared. In addition, previous study ${ }^{37}$ found that LAP was positively associated with hyperuricemia. But contrary to our results, there was not association between the LAP and hyperuricemia. The reason for the difference between this study and previous studies ${ }^{27}$ may be related to the different participant populations with different lifestyle and inclusion criteria. In this study, all the participants had normal BMI, and there were only eight participants (7 women, 1 man) with central obesity, which was an important distinction from the previous studies.

The CMI is a new anthropometric index proposed by Wakabayashi and Daimon, ${ }^{15}$ which is calculated as the product of the TG/HDL-C ratio and WHtR and appears to be a good predictor of coronary artery disease as a central component of MS. Some researchers ${ }^{38}$ have pointed out that the TG/HDL-C ratio may reflect atherogenic small dense LDL-C particles and may be associated with IR and MS; and WHtR is a better discriminator of coronary heart disease and cardiovascular risk than WC and BMI. CMI incorporates the TG/ HDL-C ratio and WHtR, and thus includes all the central components comprising the criteria of MS. In the present study, the CMI was found to be a unique anthropometric index associated with hyperuricemia, with the following possible underlying mechanisms. First, the TG/HDL-C ratio is more strongly associated with IR than with other indicators of lipid metabolism, and IR cause urate reabsorption, hyperlipemia and the subsequent overproduction of uric acid. ${ }^{33,34}$ Secondly, elevated TG levels reflected in the CMI may cause more free fatty 
Table 4 Logistic Regression Analysis for Association Between Anthropometric Indices and Hyperuricemia

\begin{tabular}{|c|c|c|c|c|c|c|c|}
\hline & \multirow[t]{2}{*}{ QI } & \multicolumn{2}{|l|}{ Q2 } & \multicolumn{2}{|l|}{ Q3 } & \multicolumn{2}{|l|}{ Q4 } \\
\hline & & OR $(95 \% \mathrm{CI})$ & $p$ values & OR $(95 \% \mathrm{Cl})$ & $p$ values & OR (95\% Cl) & $p$ values \\
\hline$A B S I$ & $\leqq 0.77$ & $0.77 \sim 0.78$ & & $0.78 \sim 0.80$ & & $>0.80$ & \\
\hline Model I & 1.000 (ref) & $0.542(0.219 \sim 1.340)$ & 0.185 & $0.502(0.220 \sim 1.144)$ & 0.101 & $0.574(0.168 \sim 1.964)$ & 0.376 \\
\hline Model 2 & 1.000 (ref) & $0.574(0.252 \sim 1.308)$ & 0.186 & $0.740(0.360 \sim 1.519)$ & $0.4 \mathrm{II}$ & $0.659(0.220 \sim 1.979)$ & 0.457 \\
\hline Model 3 & 1.000 (ref) & $0.475(0.175 \sim 1.290)$ & 0.144 & $0.321(0.110 \sim 0.935)$ & 0.037 & $0.342(0.067 \sim 1.749)$ & 0.197 \\
\hline $\mathrm{BAI}$ & $\leqq 23.20$ & $23.20 \sim 24.70$ & & $24.70 \sim 26.00$ & & $>26.00$ & \\
\hline Model I & 1.000 (ref) & $1.549(0.765 \sim 3.136)$ & 0.224 & $1.046(0.503 \sim 2.174)$ & 0.905 & $0.734(0.329 \sim 1.638)$ & 0.450 \\
\hline Model 2 & 1.000 (ref) & $\mathrm{I} .648(0.804 \sim 3.38 \mathrm{I})$ & 0.173 & $1.022(0.485 \sim 2.152)$ & 0.955 & 0.55 I (0.234 I.297) & 0.173 \\
\hline Model 3 & 1.000 (ref) & $2.204(0.969 \sim 5.015)$ & 0.059 & $\mathrm{I} .435(0.583 \sim 3.53 \mathrm{I})$ & 0.432 & $0.643(0.209 \sim 1.976)$ & 0.643 \\
\hline BRI & $\leqq 2.50$ & $2.50 \sim 2.90$ & & $2.90 \sim 3.30$ & & $>3.30$ & \\
\hline Model I & 1.000 (ref) & $0.673(0.336 \sim 1.348)$ & 0.264 & $0.657(0.3|5 \sim 1.37|)$ & 0.263 & $0.955(0.464 \sim 1.964)$ & 0.900 \\
\hline Model 2 & 1.000 (ref) & $0.675(0.333 \sim 1.365)$ & 0.274 & $0.544(0.245 \sim 1.209)$ & 0.135 & $0.66 \mathrm{I}(0.276 \sim 1.583)$ & 0.353 \\
\hline Model 3 & 1.000 (ref) & $0.972(0.372 \sim 2.539)$ & 0.953 & $0.770(0.207 \sim 2.867)$ & 0.697 & $1.255(0.269 \sim 5.848)$ & 0.773 \\
\hline VAl & $\leqq 0.80$ & $0.80 \sim 1.30$ & & $1.30 \sim 2.03$ & & $>2.03$ & \\
\hline Model I & 1.000 (ref) & $2.603(1.125 \sim 6.020)$ & 0.025 & $3.123(1.342 \sim 7.266)$ & 0.008 & $2.482(1.059 \sim 5.816)$ & 0.036 \\
\hline Model 2 & 1.000 (ref) & $2.899(1.230 \sim 6.832)$ & 0.015 & $3.36 \mathrm{I}$ (1.419 7.959) & 0.006 & $2.813(1.158 \sim 6.832)$ & 0.022 \\
\hline Model 3 & 1.000 (ref) & $1.920(0.75 \mathrm{I} \sim 4.907)$ & 0.173 & $1.588(0.601 \sim 4.198)$ & 0.351 & $\mathrm{I} .688(0.639 \sim 4.46 \mathrm{I})$ & 0.291 \\
\hline LAP & $\leqq 12.66$ & $12.66 \sim 19.55$ & & $19.55 \sim 28.69$ & & $>28.69$ & \\
\hline Model I & 1.000 (ref) & I. $147(0.554 \sim 2.375)$ & 0.711 & $0.87 \mid(0.406 \sim 1.867)$ & 0.723 & $1.163(0.562 \sim 2.408)$ & 0.684 \\
\hline Model 2 & 1.000 (ref) & I.099 (0.5। 4 2.349) & 0.808 & $0.914(0.420 \sim 1.989)$ & 0.821 & $\mathrm{I} .263(0.580 \sim 2.75 \mathrm{I})$ & 0.557 \\
\hline Model 3 & 1.000 (ref) & $1.125(0.468 \sim 2.707)$ & 0.792 & $0.520(0.206 \sim 1.312)$ & 0.166 & $0.709(0.268 \sim 1.872)$ & 0.487 \\
\hline CMI & $\leqq 0.26$ & $0.26 \sim 0.39$ & & $0.39 \sim 0.64$ & & $>0.64$ & \\
\hline Model I & 1.000 (ref) & $3.958(1.053 \sim 14.878)$ & 0.042 & 6.991 (1.976 24.737) & 0.003 & 24.359 (7.184 82.591) & $<0.001$ \\
\hline Model 2 & 1.000 (ref) & $4.232(I .121 \sim 15.981)$ & 0.033 & 7.349 (2.067 26.123) & 0.002 & 25.684 (7.527 87.642) & $<0.001$ \\
\hline Model 3 & 1.000 (ref) & $3.540(0.875 \sim 14.326)$ & 0.076 & $5.488(1.403 \sim 21.472)$ & 0.013 & $16.674(4.424 \sim 62.846)$ & $<0.001$ \\
\hline
\end{tabular}

Notes: Model I was crude, Model 2 adjusted for age and gender: Model 3 adjusted for age, gender, BMI and biochemical indicators (FPG, urea, SCr, TC and LDL-C).

Table 5 ROC Curve of Different Anthropometric Indices Predictive Value for Hyperuricemia

\begin{tabular}{|l|c|c|c|c|c|c|c|c|}
\hline Variable & AUC & Youden & Cutoff Value & Sensitivity & Specificity & \multicolumn{2}{|c|}{$\mathbf{9 5 \%}$ CI } & \multirow{2}{*}{ value } \\
\cline { 4 - 8 } & & & & & & & Lower & Upper \\
\hline CMI & 0.777 & $4 I .4 \%$ & 0.655 & $57.1 \%$ & $84.2 \%$ & 0.719 & 0.835 & $<0.001$ \\
LAP & 0.501 & $5.9 \%$ & 22.260 & $43.1 \%$ & $62.9 \%$ & 0.425 & 0.577 & 0.985 \\
VAI & 0.592 & $21.1 \%$ & 0.950 & $85.7 \%$ & $35.4 \%$ & 0.525 & 0.658 & 0.017 \\
ABSI & 0.507 & $7.5 \%$ & 0.815 & $18.6 \%$ & $89.0 \%$ & 0.430 & 0.585 & 0.852 \\
BAI & 0.525 & $10.0 \%$ & 25.85 & $28.6 \%$ & $83.4 \%$ & 0.453 & 0.570 & 0.515 \\
BRI & 0.521 & $9.1 \%$ & 2.45 & $80.5 \%$ & $28.6 \%$ & 0.441 & 0.601 & 0.590 \\
\hline
\end{tabular}

acids to be produced and utilized, and the synthesis of fatty acids in the liver is associated with the de novo synthesis of purines, which accelerate uric acid production. ${ }^{39,40}$ The last and possibly most important reason explaining the association between the CMI and hyperuricemia is that low HDL-C levels are independently associated with decreased eGFR and increased risk of renal impairment, which may result in decreased excretion of uric acid. ${ }^{41-43}$

This study has several limitations. First, the study was cross-sectional in design, which prevented us from investigating the causal association between CMI and SUA levels and the association between blood biomarkers, anthropometric indices, and the progression of hyperuricemia. 


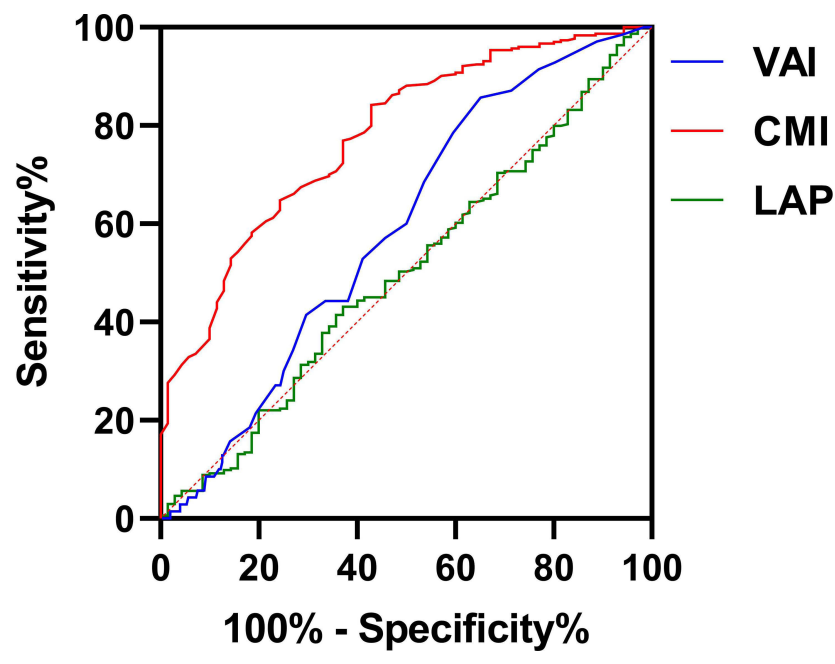

Figure 2 The predictive value of $\mathrm{CMI}$ and other anthropometric indices for hyperuricemia.

Secondly, the sample size was small, and all the participants were enrolled from one urban region with similar lifestyles and eating habits, so our power to investigate deeper relationships or findings was limited. Thirdly, we did not stratify the participants by gender, and neither was the menopausal status of females, the difference in the consumption of foods with high purine content, alcohol and soft drink sweetened with fructose collected, which are the main modifiable variables related to hyperuricemia development.

In conclusion, the CMI showed a moderate positive association with hyperuricemia, and offered higher predictive values for hyperuricemia. CMI can be easily obtained in annual check-up examinations and can provide monitoring bioindicators for hyperuricemia management of asymptomatic populations with normal BMI.

\section{Key-Messages}

Hyperuricemia is highly prevalent in asymptomatic population with normal BMI in China.

CMI was moderate positive association with serum uric acid level, was independently associated with hyperuricemia.

CMI can offer higher predictive values for hyperuricemia, which can easily obtain in check-up examinations.

\section{Acknowledgment}

We are grateful to Xing Guan, Yan-Feng Li for providing technical assistance.

\section{Disclosure}

The authors declare that they have no conflicts of interest.

\section{References}

1. Maloberti A, Qualliu E, Occhi L, et al. Hyperuricemia prevalence in healthy subjects and its relationship with cardiovascular target organ damage. Nutr Metab Cardiovasc Dis. 2021;31:178-185. doi:10.1016/ j.numecd.2020.08.015

2. Dehlin M, Jacobsson L, Roddy E. Global epidemiology of gout: prevalence, incidence, treatment patterns and risk factors. Nat Rev Rheumatol. 2020;16:380-390. doi:10.1038/s41584-020-0441-1

3. Liu XZ, Li HH, Huang S, Zhao DB. Association between hyperuricemia and nontraditional adiposity indices. Clin Rheumatol. 2019;38:1055-1062. doi:10.1007/s10067-018-4374-x

4. Krakauer NY, Krakauer JC, Li S. A new body shape index predicts mortality hazard independently of body mass index. PLoS One. 2012;7:e39504. doi:10.1371/journal.pone.0039504

5. Bergman RN, Stefanovski D, Buchanan TA, et al. A better index of body adiposity. Obesity (Silver Spring). 2011;19(5):1083-1089. doi:10.1038/oby.2011.38

6. Thomas DM, Bredlau C, Bosy-Westphal A, et al. Relationships between body roundness with body fat and visceral adipose tissue emerging from a new geometrical model. Obesity (Silver Spring). 2013;21(11):2264-2271. doi:10.1002/oby.20408

7. Rospleszcz S, Dermyshi D, Müller-Peltzer K, Strauch K, Bamberg F, Peters A. Association of serum uric acid with visceral, subcutaneous and hepatic fat quantified by magnetic resonance imaging. Sci Rep. 2020;10:442. doi:10.1038/s41598-020-57459-Z

8. Zong J, Sun Y, Zhang Y, et al. Correlation between serum uric acid level and central body fat distribution in patients with type 2 diabetes. Diabetes Metab Syndr Obes. 2020;13:2521-2531. doi:10.2147/ DMSO.S260891

9. Amato MC, Giordano C, Galia M, et al.; AlkaMeSy Study Group. Visceral adiposity index: a reliable indicator of visceral fat function associated with cardiometabolic risk. Diabetes Care. 2010;33 (4):920-922. doi:10.2337/dc09-1825

10. Huang X, Jiang X, Wang L, et al. Visceral adipose accumulation increased the risk of hyperuricemia among middle-aged and elderly adults: a population-based study. J Transl Med. 2019;17:341. doi:10.1186/s12967-019-2074-1

11. Bawadi H, Abouwatfa M, Alsaeed S, Kerkadi A, Shi Z. Body shape index is a stronger predictor of diabetes. Nutrients. 2019;11:1018. doi:10.3390/nu11051018

12. Rico-Martín S, Calderón-García JF, Sánchez-Rey P, Franco-Antonio C, Martínez Alvarez M, Sánchez Muñoz-Torrero JF. Effectiveness of body roundness index in predicting metabolic syndrome: a systematic review and meta-analysis. Obes Rev. 2020;21:e13023. doi:10.1111/ obr. 13023

13. Biyik Z, Guney I. Lipid accumulation product and visceral adiposity index: two new indices to predict metabolic syndrome in chronic kidney disease. Eur Rev Med Pharmacol Sci. 2019;23:2167-2173. doi:10.26355/eurrev_201903_17262

14. Zhang X, Hong F, Liu L, et al. Lipid accumulation product is a reliable indicator for identifying metabolic syndrome: the China Multi-Ethnic Cohort (CMEC) Study. QJM. 2020;24:hcaa325. doi:10.1093/qjmed/hcaa325

15. Wakabayashi I, Daimon T. The "cardiometabolic index" as a new marker determined by adiposity and blood lipids for discrimination of diabetes mellitus. Clin Chim Acta. 2015;438:274-278. doi:10.1016/j. cca.2014.08.042

16. Multidisciplinary Expert Task Force on Hyperuricemia and Related Diseases. Chinese multidisciplinary expert consensus on the diagnosis and treatment of hyperuricemia and related diseases. Chin Med $J$ (Engl). 2017;130(20):2473-2488. doi:10.4103/0366-6999.216416

17. Sam S. Differential effect of subcutaneous abdominal and visceral adipose tissue on cardiometabolic risk. Horm Mol Biol Clin Investig. 2018;33. doi:10.1515/hmbci-2018-0014 
18. Kuo KL, Chen HM, Hsiao SH, et al. The relationship between anthropometric factors and hyperuricemia in adolescent athletes. Obes Res Clin Pract. 2021;15:375-380. doi:10.1016/j. orcp.2021.03.012

19. Chaudry O, Grimm A, Friedberger A, et al. Magnetic resonance imaging and bioelectrical impedance analysis to assess visceral and abdominal adipose tissue. Obesity (Silver Spring). 2020;28:277-283. doi:10.1002/oby.22712

20. Nusrianto R, Tahapary DL, Soewondo P. Visceral adiposity index as a predictor for type 2 diabetes mellitus in Asian population: a systematic review. Diabetes Metab Syndr. 2019;13:1231-1235. doi:10.1016/j.dsx.2019.01.056

21. Klisić A, Kavarić N, Spasojević-Kalimanovska V, Kotur-Stevuljević J, Ninić A. Serum endocan levels in relation to traditional and non-traditional anthropometric indices in adult population. $J$ Med Biochem. 2021;40:41-48. doi:10.5937/jomb0-25170

22. Gu Z, Zhu P, Wang Q, et al. Obesity and lipid-related parameters for predicting metabolic syndrome in Chinese elderly population. Lipids Health Dis. 2018;17:289. doi:10.1186/s12944-018-0927-x

23. Gasevic D, Frohlich J, Mancini GJ, Lear SA. Clinical usefulness of lipid ratios to identify men and women with metabolic syndrome: a cross-sectional study. Lipids Health Dis. 2014;13:159. doi:10.1186/ 1476-511X-13-159

24. Pantoja-Torres B, Toro-Huamanchumo CJ, Urrunaga-Pastor D, et al. Insulin resistance and metabolic syndrome research group high triglycerides to HDL-cholesterol ratio is associated with insulin resistance in normal-weight healthy adults. Diabetes Metab Syndr Clin Res Rev. 2018;13:382-388. doi:10.1016/j.dsx.2018.10.006

25. Hurrle S, Hsu WH. The etiology of oxidative stress in insulin resistance. Biomed J. 2017;40:257-262. doi:10.1016/j.bj.2017.06.007

26. Yaribeygi H, Farrokhi FR, Butler AE, Sahebkar A. Insulin resistance: review of the underlying molecular mechanisms. J Cell Physiol. 2019;234(6):8152-8161. doi:10.1002/jcp.27603

27. Gu D, Ding Y, Zhao Y, Miao S, Qu Q. Positively increased visceral adiposity index in hyperuricemia free of metabolic syndrome. Lipids Health Dis. 2018;17:101. doi:10.1186/s12944-018-0761-1

28. Krajcoviechova A, Tremblay J, Wohlfahrt P, et al. The impact of blood pressure and visceral adiposity on the association of serum uric acid with albuminuria in adults without full metabolic syndrome. Am J Hypertens. 2016;29:1335-1342. doi:10.1093/ajh/hpw098

29. Ahn N, Baumeister SE, Amann U, et al. Visceral adiposity index (VAI), lipid accumulation product (LAP), and product of triglycerides and glucose (TyG) to discriminate prediabetes and diabetes. Sci Rep. 2019;9:9693. doi:10.1038/s41598-019-46187-8

30. Dong $\mathrm{H}, \mathrm{Xu} \mathrm{Y}$, Zhang $\mathrm{X}$, Tian S. Visceral adiposity index is strongly associated with hyperuricemia independently of metabolic health and obesity phenotypes. Sci Rep. 2017;7:8822. doi:10.1038/s41598-01709455-z

31. Muscelli E, Natali A, Bianchi S, et al. Effect of insulin on renal sodium and uric acid handling in essential hypertension. $\mathrm{Am}$ J Hypertens. 1996;9:746-752. doi:10.1016/0895-7061(96)00098-2
32. Perez-Ruiz F, Aniel-Quiroga MA, Herrero-Beites AM, Chinchilla SP, Erauskin GG, Merriman T. Renal clearance of uric acid is linked to insulin resistance and lower excretion of sodium in gout patients. Rheumatol Int. 2015;35:1519-1524. doi:10.1007/s00296-015-3242-0

33. Bjornstad P, Eckel RH. Pathogenesis of lipid disorders in insulin resistance: a brief review. Curr Diab Rep. 2018;18:127. doi:10.1007/s11892-018-1101-6

34. Liu XY, Wu QY, Chen ZH, et al. Elevated triglyceride to high-density lipoprotein cholesterol (TG/HDL-C) ratio increased risk of hyperuricemia: a 4-year cohort study in China. Endocrine. 2020;68:71-80. doi:10.1007/s12020-019-02176-5

35. Redon P, Maloberti A, Facchetti R, et al. Gender-related differences in serum uric acid in treated hypertensive patients from central and east European countries: findings from the Blood Pressure control rate and CArdiovascular Risk profilE study. $J$ Hypertens. 2019;37:380-388. doi:10.1097/HJH.0000000000001908

36. Pugliese NR, Mengozzi A, Virdis A, et al.; Working Group on Uric Acid and Cardiovascular Risk of the Italian Society of Hypertension. The importance of including uric acid in the definition of metabolic syndrome when assessing the mortality risk. Clin Res Cardiol. 2021;110:1073-1082. doi:10.1007/s00392-021-01815-0

37. Seong JM, Park CE, Gi MY, et al. Relationship between uric acid and lipid accumulation product index by gender in Korean adults: the 2016 Korean National Health and Nutrition Examination Survey. Prim Care Diabetes. 2021;15:541-547. doi:10.1016/j.pcd.2020.12.001

38. Jeppesen J, Hein HO, Suadicani P, Gyntelberg F. Relation of high TG-low HDL cholesterol and LDL cholesterol to the incidence of ischemic heart disease. An 8-year follow-up in the Copenhagen Male Study. Arterioscler Thromb Vasc Biol. 1997;17:1114-1120. doi:10.1161/01.atv.17.6.1114

39. Matsuura F, Yamashita S, Nakamura T, et al. Effect of visceral fat accumulation on uric acid metabolism in male obese subjects: visceral fat obesity is linked more closely to overproduction of uric acid than subcutaneous fat obesity. Metabolism. 1998;47:929-933. doi:10.1016/s0026-0495(98)90346-8

40. Balasubramanian T. Uric acid or 1-methyl uric acid in the urinary bladder increases serum glucose, insulin, true triglyceride, and total cholesterol levels in Wistar rats. ScientificWorldJournal. 2003;3:930-936. doi:10.1100/tsw.2003.90

41. Kawachi K, Kataoka H, Manabe S, Mochizuki T, Nitta K. Low HDL cholesterol as a predictor of chronic kidney disease progression: a cross-classification approach and matched cohort analysis. Heart Vessels. 2019;34:1440-1455. doi:10.1007/s00380-019-01375-4

42. Wang F, Zheng J, Ye P, et al. Association of high-density lipoprotein cholesterol with the estimated glomerular filtration rate in a community-based population. PLoS One. 2013;8:e79738. doi:10.1371/journal.pone.0079738

43. Wright AF, Rudan I, Hastie ND, Campbell H. A 'complexity' of urate transporters. Kidney Int. 2010;78:446-452. doi:10.1038/ ki.2010.206
International Journal of General Medicine

\section{Publish your work in this journal}

The International Journal of General Medicine is an international, peer-reviewed open-access journal that focuses on general and internal medicine, pathogenesis, epidemiology, diagnosis, monitoring and treatment protocols. The journal is characterized by the rapid reporting of reviews, original research and clinical studies across all disease areas. The manuscript management system is completely online and includes a very quick and fair peer-review system, which is all easy to use. Visit http://www.dovepress.com/ testimonials.php to read real quotes from published authors. 